\title{
Malignant Peritoneal Mesothelioma: A Case Report
}

Purushotham Reddy ${ }^{1}$, Ravi Koppad, Nayantara M. Nirgude ${ }^{3 *}$

${ }^{1}$ Professor and Head of Department of Pathology, Karnataka Institute of Medical Sciences, PB Rd, Vidya Nagar, Hubli, Karnataka 580022, India

${ }^{2}$ Assistant professor, Department of Surgical Oncology, Karnataka Institute of Medical Sciences, PB Rd, Vidya Nagar, Hubli, Karnataka 580022, India

${ }^{3}$ Department of Pathology, Karnataka Institute of Medical Sciences, PB Rd, Vidya Nagar, Hubli, Karnataka 580022, India

DOI: $\underline{10.36348 / \mathrm{sjpm} .2022 . \mathrm{v} 07 \mathrm{i} 01.001}$

| Received: 04.12.2021 | Accepted: 06.01.2022 | Published: 08.01.2022

*Corresponding author: Dr. Nayantara M. Nirgude

Department of Pathology, Karnataka Institute of Medical Sciences, PB Rd, Vidya Nagar, Hubli, Karnataka 580022, India

\section{Abstract}

Mesothelioma of peritoneum is a rare malignancy of serosal membranes. Here we report a case of 55year old female with mass per abdomen reported as epithelioid type of peritoneal mesothelioma. The diagnostic challenge in our cases was due to non specific presentation and the varied histologic morphology of tumor cells for which immunohistochemical studies with markers calretinin, vimentin, cytokeratin 20, cytokeratin5/6, CD34 and HMB34 had to be performed to arrive at the diagnosis. The tumor cells showed positvity for calretinin, vimentin and cytokeratin 20 . The final diagnosis was possible due to correlation of histological and immunohistochemical characteristics been consistent with mesothelial origin.

Keywords: Mesothelioma, Peritoneum, Malignant, Calretinin, Cytokeratin 20, And Vimentin.

Copyright (C) 2022 The Author(s): This is an open-access article distributed under the terms of the Creative Commons Attribution 4.0 International License (CC BY-NC 4.0) which permits unrestricted use, distribution, and reproduction in any medium for non-commercial use provided the original author and source are credited.

\section{INTRODUCTION}

Mesothelioma is a very rare malignancy of serosal membranes arising from pleura, peritoneum, pericardium and tunica vaginalis testes. Peritoneal mesothelioma nearly accounts for $7-30 \%$ of malignant mesotheliomas. The peritoneum is second most common site of mesothelium affected the following the pleura. Malignant peritoneal mesothelioma is difficult to diagnose due to its vague, nonspecific symptoms. It commonly presents with diffuse, extensive spread throughout the abdomen with rare metastatic spread beyond the abdominal cavity. The contact with asbestos is the most significant risk factor for malignant pleural mesothelioma but the relationship with malignant mesothelioma of other cavities is not as well [1]. Specifically in women with malignant peritoneal mesothelioma, causality of asbestos is not clear and many patients have no known asbestos exposure [2].

\section{CASE REPORT}

A 55year old female was operated for umbilical hernia and omental biopsy work up showed adenocarcinoma deposits. The PET CT reports of the patient were inconclusive. And 4 months later the patient presented with mass per abdomen which was arising from parietal wall and this mass advanced quickly in a month duration. Ileocolic resection with total abdominal hysterectomy was done. Operative findings showed a mass involving ileocolic region in continuation with parietal wall with multiple jejunal wall and pelvic peritoneal deposits. On gross examination the ileocolic segment of intestine showed a mass with entangled coils of intestine with adherent omental fat, part of abdominal wall and skin mass measuring $20 \times 12 \times 12 \mathrm{cms}$ with skin flap with umbilicus measuring $4 \times 1 \mathrm{~cm}$. Cut surface showed an irregular white tumor with blackish red areas in intestine extending to serosa, mesentry, subcutaneous tissue and rectus muscle of abdominal wall. The jejunal resection specimen showed serosal and mesenteric mass largest mass measuring $4 \times 3 \times 3 \mathrm{cms}$. External surface showed blackish discolouration. On cut surface, a soft haemorrhagic mass with focal white areas seen extending to the mucosa and muscular layer of small intestine. The uterus on cut surface showed a fibroid measuring $2 \mathrm{~cm}$ in diameter, the bilateral fallopian tubes and ovaries were unremarkable.

On microscopy the tumor showed round to oval cells in sheets with pleomorphic nuclei with coarse chromatin and prominent nucleoli and eosinophilic to 
Purushotham Reddy et al.; Saudi J Pathol Microbiol, Jan, 2022; 7(1): 1-3

clear cytoplasm. Tumour cells appeared to be forming an irregular vascular channels with lakes of red blood cells and at focal areas capillaries were lined by atypical endothelial lining (Fig 1). Mitotic figures were seen frequently. Tumour was seen involving the full thickness of both small and large intestine including the mucosa of intestine and mesentery along with subcutaneous tissue. Uterus, cervic, both fallopian tubes and ovaries were normal except for fibroid. The above features of intestinal and serosal mass gave a differential diagnosis of epithelioid mesothelioma and epithelioid angiosarcoma. Following which immunohistochemical study was done using markers calretinin, cytokeratin 20 vimentin, cytokeratin5/6, HMB-45 and CD 34. The tumour cells showed positivity for calretinin, cytokeratin $5 / 6$ and Vimentin and negative for all other markers used, suggesting epithelioid malignant mesothelioma (Fig 2).

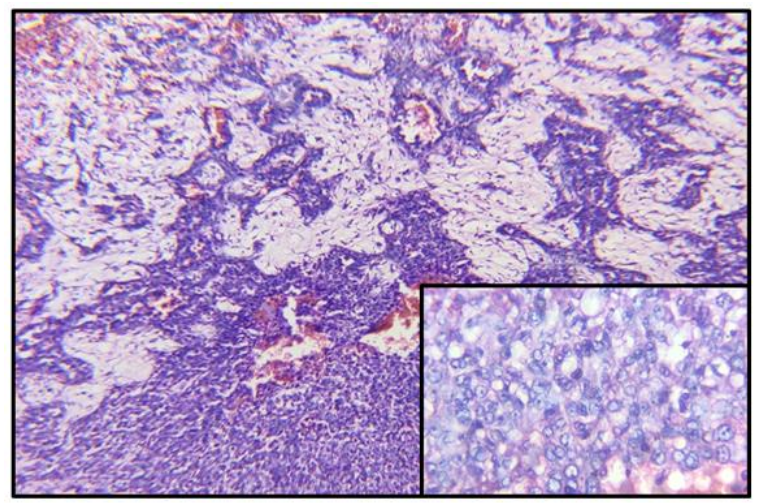

Fig 1: Tumour cells appeared to be forming an irregular vascular channels with lakes of red blood cells and at focal areas capillaries were lined by atypical endothelial lining

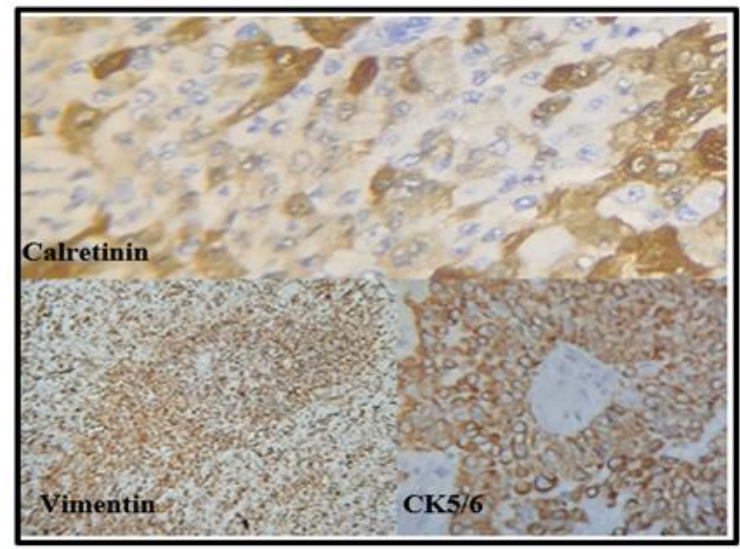

Fig 2: Tumour cells showing positivity for calretinin, cytokeratin5/6 and vimentin

\section{DISCUSSION}

Mesothelioma is a tumour originating from mesothelial cells lining the serous cavities. It has previously been established that cumulative asbestos exposure increases the risk of mesothelioma by a corresponding amount. Mesothelioma can be caused by non-industrial environmental exposure to asbestos fibres, as well as para-occupational exposure. Several non-asbestos aetiologies, such as therapeutic irradiation, chronic inflammatory peritonitis and simian virus-40, are carcinogenic.

As the presentation of the patient is nonspecific, leading to delayed and difficult diagnosis [4]. De pangher manzini et al., reported the most common presenting symptoms were ascites and abdominal pain followed by weakness, weight loss, anorexia, abdominal mass, fever, diarrhoea and vomiting [5]. In our case the patient had presented with mass per abdomen. According to Kurodo et al mesothelioma of mesentry almost presents all time as thickening of peritoneal surface and the localised mass of mesentry may case luminal obstruction of the bowel [6].

Malignant peritoneal mesothelioma is classified into different histological types: epithelioid, sarcomatous and biphasic. The most common presentation is epithelioid type. On histology the epithelioid mesothelioma subtype shows cells arranged in tubulopapillary, micro glandular, solid small cell and pleomorphic. The connective tissue subtype mesothelioma can be fibroblastic, muscle like, cartilaginous, osseous, angiomatoid and fibrous. In our case the cell morphology was of epithelioid type but focal areas showed angiomatoid arrangement [7]. The vasoformative pattern of arrangement with epithelioid type of cells also pointed towards angiosarcoma as diagnosis [8]. Immunohistochemical studies was suggested for the case. And with current recommendations two mesothelial specific markers and two epithelial specific markers were used [9]. Immunohistochemical study was performed with stains calretinin, cytokeratin 5/6, Vimentin and cytokeratin 20 . Additionally, CD34, HMB-45, synaptophysin and chromogranin immunohistochemical markers were used to know if the cell of origin was endothelial in nature, neuroendocrine origin or melanocytic nature. The tumour cells showed calretinin, cytokeratin5/6 and vimentin positivity while others were negative favouring the diagnosis of epithelioid type of malignant mesothelioma.

Malignant peritoneal mesothelioma is therapeutically treated with cytoreductive studies and hyper thermic intraperitoneal chemotherapy. The prognosis depends on the histologic type. The Epithelioid subtype mesothelioma has better prognosis than sarcomatoid or biphasic type [10].

\section{CONCLUSION}

Malignant peritoneal mesothelioma is a rare malignant neoplasm. The diagnosis of which is challenging considering the non-specific presentation of patient and varied histomophology leading to delay in 
diagnosis. Definitive diagnosis has to be made based on both histologic and Immunohistochemical studies.

\section{REFERENCES}

1. Kim, J., Bhagwandin, S., \& Labow, D. M. (2017). Malignant peritoneal mesothelioma: a review. Annals of translational medicine,5(11), 236.

2. Zha, B. S., Flanagan, M., Coulson, C., \& Garvin, K. W. (2015). Difficult to Identify: Malignant Primary Peritoneal Mesothelioma. The American journal of medicine, 128(11), 1191-1194.

3. Algın, M. C., Yaylak, F., Bayhan, Z., Aslan, F., \& Bayhan, N. A. (2014). Malignant peritoneal mesothelioma: clinicopathological characteristics of two cases. Case reports in surgery, 2014, 748469.

4. Ibrahim, A. M., Al-Akchar, M., Obaidi, Z., \& AlJohany, H. (2018). Malignant Peritoneal Mesothelioma: A Rare Cause of Ascites. Journal of investigative medicine high impact case reports, 6 , 2324709618807506.

5. Manzini, V. P., Recchia, L., Cafferata, M., Porta, C., Siena, S., Giannetta, L., Morelli, F., Oniga, F., Bearz, A., Torri, V., \& Cinquini, M. (2010).
Malignant peritoneal mesothelioma: a multicenter study on 81 cases. Annals of oncology: official journal of the European Society for Medical Oncology, 21(2), 348-353.

6. Bani-Hani, K. E., \& Gharaibeh, K. A. (2005). Malignant peritoneal mesothelioma. Journal of surgical oncology, 91(1), 17-25.

7. Attanoos, R. L., \& Gibbs, A. R. (1997). Pathology of malignant mesothelioma. Histopathology, 30(5), 403-418.

8. Usuda, H., \& Naito, M. (1997). Multicentric angiosarcoma of the gastrointestinal tract. Pathology international, 47(8), 553-556.

9. Chapel, D. B., Schulte, J. J., Husain, A. N., \& Krausz, T. (2020). Application of immunohistochemistry in diagnosis and management of malignant mesothelioma. Translational lung cancer research, 9(Suppl 1), S3-S27.

10. Frontario, S. C., Loveitt, A., Goldenberg-Sandau, A., Liu, J., Roy, D., \& Cohen, L. W. (2015). Primary Peritoneal Mesothelioma Resulting in Small Bowel Obstruction: A Case Report and Review of Literature. The American journal of case reports, 16, 496-500. 